\title{
New Approaches to E-Cognitive Assessment of E-Learning
}

\author{
Morales-Martinez Guadalupe Elizabeth ${ }^{1,2}$, Lopez-Ramirez Ernesto Octavio ${ }^{3}$, Lopez-Gonzalez \\ Ana Elizabeth ${ }^{2}$ \\ Institute of Research on the University and Education (IISUE) $7^{\text {th } 1}$ \\ National Autonomous University of Mexico (UNAM) ${ }^{2}$ \\ Nuevo Leon Autonomous University $(U A N L)^{3}$
}

\begin{abstract}
A technology transfer project to innovate $e$ cognitive assessment is described. Specifically, natural semantic networks (as opposed to idiosyncratic or artificial semantic nets) from 880 high school and bachelor students and teachers from different knowledge domains were used to computer simulate schemata behavior regarding each domain. Schemata-related words were implemented in a semantic priming study that students had to take before and after a course. Then a neural net was implemented capable of discriminating between successful and unsuccessful students by analyzing students' recognition times to schemata-related words. A computer system was developed to use these research results to empower teachers with reports of schema development, meaning formation indexes and neural net classification due to learning. It is argued that this project should point to new alternatives assess e-learning. The idea is not to substitute current standard assessment but to use computer science advances to innovate assessment of e-learning.
\end{abstract}

\section{Introduction}

Educational Information and Communication Technologies (ICT) have proved to be an excellent resource when they are properly introduced into educational settings. For instance, since the introduction of computer-assisted instruction systems (PLATO, TICCIT, Intelligent Computer-Assisted Instruction (ICAI), guided learning systems, etc. ${ }^{1}$ ) up to recent virtual learning platforms like Learning Space, Blackboard, Moodle, etc. (systems to enhance connectivity, group discussions, collaborative and cooperative learning, etc [2], [3]), there has been an amazing development of instruction models that facilitate e-learning. Thus, development of interfaces (with high usability level) that empower learning with sophisticated multimedia presentations [4], as well as provide access to digital bases, semantic web [5], and augmented reality [6], [7] are by now some examples of how e-learning and e-instruction redefine themselves constantly toward a modern education era. However, this solid updating of education technology is not true if e-learning assessment is considered. Rather, standard methods to evaluate school learning have been constantly adapted and used to evaluate students' e-learning (standard testing, learning activities, products and even group discussion Aristotelian tasks). There is a tremendous gap between e-instruction innovation and e-learning assessment innovation that cannot be ignored any more. We cannot continue fixing, patching or readapting the past into the new digital classroom.

Here, it is argued that by considering new advances in computer science as well as cognitive science methods to determine memory concept organization and mental representation, it is possible to implement a new empirical direction to innovate e-cognitive assessment of e-learning. Specifically, a system is presented capable of training neural networks to identify whether students have integrated into their lexicon new concepts that are related by a schema knowledge taught in a classroom [8] Here, neural nets are trained to discriminate between successful and unsuccessful students' semantic priming latencies of schemata-related words obtained by a semantic priming study at the beginning and the end of a course. This neural network discrimination capacity is based on the idea that once a student has integrated new knowledge into long term memory then a semantic priming effect is obtained from schemata-related words (e.g., single word schemata priming [9]. This system is named EVCO (Cognitive Evaluator in Spanish) and empirical evidence supporting its development is presented next.

The EVCO system is currently an education technology transfer project carried over in Mexico. A prototype has been developed and now is in the process of testing its functionality in different online educational settings throughout the country. Let us first to describe the methodology used to implement the prototype and then discuss how this prototype is implemented as an education transfer technology project. 


\section{Method}

The prototype implementation was developed by following three different research phases. First, computer software was developed capable of obtaining mental representations of course contents from teachers and students before and after a course. Here, a computer system was developed capable of providing around 20 indices underlying meaning formation and schemata concept organization. In a second phase, the obtained representations of a course content were allowed to carry over computer simulations of schemata behavior based on teachers' and students' semantic nets. In turn these simulations allowed researchers to obtain word pairs related by schemata behavior for each knowledge domain.

Then students were required to take semantic priming studies before and after the course, where recognition times to schemata words from their respective courses were compared against standard semantic-related word pairs' response times. In a third phase a neural net was trained to discriminate between successful and unsuccessful response times to schemata-related words.

\subsection{First Phase}

In order to obtain a mental representation tool capable of providing semantic indices of concept organization, a technique called natural semantic networks tested as the most suitable among several others to simulate schemata behavior in the current project. Generally speaking, in a natural semantic network study participants are required to define target concepts which are related by a schema. Here, target concepts are provided by teachers and experts on the schema to be learned. Students have to define target concepts by using other single concepts (definers). The ten highest ranked definers (SAM group) for each target concept are obtained before and after a course and are taken into account to evaluate differences in concept organization due to a course. Some concepts serve as definers for more than one target concept. These concepts are called common definers and groups of definers are interconnected through them. This technique has been tested [10], [11], [12] and shown to produce definitions for the represented objects based on their meaning and not on free associations or pure semantic category membership [9].

Natural semantic nets can be drawn if desired where common definers serve as links between target concepts and SAM groups. Figure 1 (middle panel) shows some psychology bachelor students' definitions of target concepts after a course on Piaget's theory. Meaning formation underlying the schema to be learned is not analyzed only by qualitative observations of definers but by a set of five semantic organization indexes. A natural semantic network of concepts is typified by a smallworld structure/scale-free network containing the combination of highly clustered neighborhoods and a short average path length where a relatively small number of well-connected nodes serve as hubs, and the distribution of node connectivity follows a power function (see Figure 1, right panel [13]). Growing and development through time can be determined in these kinds of nets [14]. In addition, statistics and network metrics can be computed from the net [15].

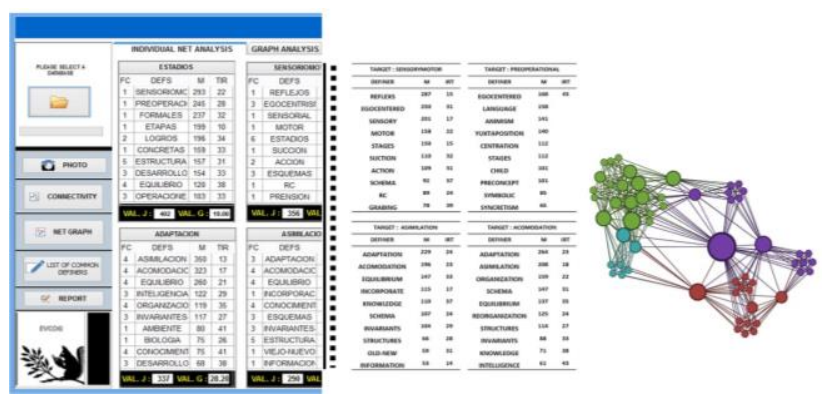

Figure 1. A natural semantic network

Left panel shows a computer screen output from a natural semantic net analysis. The middle panel shows English translation to four different conceptual definition groups for concepts regarding Piaget's theory. The M value represents students ranking whereas the inter-response time represents the average time a definer tends to appear in a group. The right panel shows the small world/scale free network structure implied by a natural semantic network (GEPHI visualization).

Around 800 hundred students from different knowledge domains (biology, psychology, music, moral development, business, etc.) provided conceptual definitions to schema concepts regarding their courses. These semantic nets were used to simulate schemata behavior as follows.

\subsection{Second Phase}

A computer system was implemented to simulate human schemata behavior according to Rumelhart et al.'s model [16]. Specifically, a constraint satisfaction neural net (Boltzmann machine) used a weight association matrix among concepts where the probability that two concept definers co-occur through SAM groups follows to:

$$
\begin{gathered}
\text { Wij }=-\ln [p(X=0 \& Y=1) p(X=1 \& Y=0)][p(X=1 \& \\
Y=1) p(X=0 \& Y=0)]-1
\end{gathered}
$$

where $\mathrm{X}$ represents one of the pair of concepts to be associated, and $\mathrm{Y}$ another concept.

For association values among concepts in a natural semantic network like the one appointed 
before, the joint probability value $\mathrm{P}(\mathrm{X}=1 \& \mathrm{Y}=0)$ can be obtained by computing how many times the definer $\mathrm{X}$ of a pair of concepts appeared in a list of definers in which Y did not appear, and the same for the other probability values. Lopez and Theios [17] and Lopez [18] have shown that this adaptation of a natural semantic network to Rumelhart et al. model does produce schemata concept organization. Figure 2 shows an example of this adaptation where the left panel relates to a computer screen output from a computer simulation of students' schema of a course on moral development. Note that if the "parents" concept is activated the "police" concept is also activated, revealing that these high school students remain in what Piaget calls a Heteronymous moral stage. The right panel in Figure 2 shows that this stage is reflected in a surface plot of the weight association matrix since the concept police obtained the highest matrix association values.

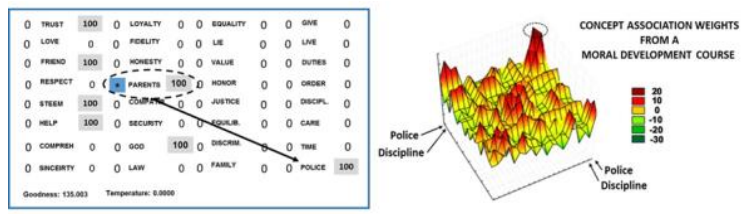

Figure 2. A connectionist simulated schema on moral development (left) as well as the weight association matrix among concepts (with authors permision ) [9].

By manipulation of concept activity (e.g., clamping concepts), it is possible to know schematarelevant concepts [18], [19], [20]. If schema-related word pairs are selected and tested in a semantic priming experiment then schemata-related word pairs seem to be recognized as significantly different from categorically related, associative related and nonrelated word pairs. Lopez called this effect schemata priming [18], [20], [21].

Several studies through different knowledge domains provide evidence that successful students of a course present schemata priming only after a course [9]. Since schemata priming is assumed to be an effect due to long-term integration of information in the lexicon, the appointed EVCO system takes advantage of this effect to train a neural network to recognize this effect. The goal was to implement a neural net capable of discriminating between successful and unsuccessful students by using the schema priming effect as follows.

\subsection{Third phase}

In order to obtain a neural net classifier a set of classification simulations was carried out. To this purpose the Neuro Solutions PRO V7 was used to implement our first successful classifier, which consisted of a supervised feed forward three-layered neural net with back propagation error. The input layer consisted of 10 units, the hidden layer had 21 units and the output layer had another set of 10 units (see top panel Figure 3).

The bottom panel of Figure 3 shows the basic idea used to build this classifier. Input neurons codified not only the schema-related word pairs but associative and nonrelated word pairs. A group of input nodes is designed to codify if the provided information belongs to a successful or to an unsuccessful student during the neural net training phase. During training, the neural net needs to be presented with around 3530 exemplars to converge on a desired classification performance. Different acceptable outcome performances are obtained from this classifier, and through manipulation of its genetic algorithm parameters, it is possible to obtain a $98 \%$ correct classification of students ${ }^{\prime}$ profiles.

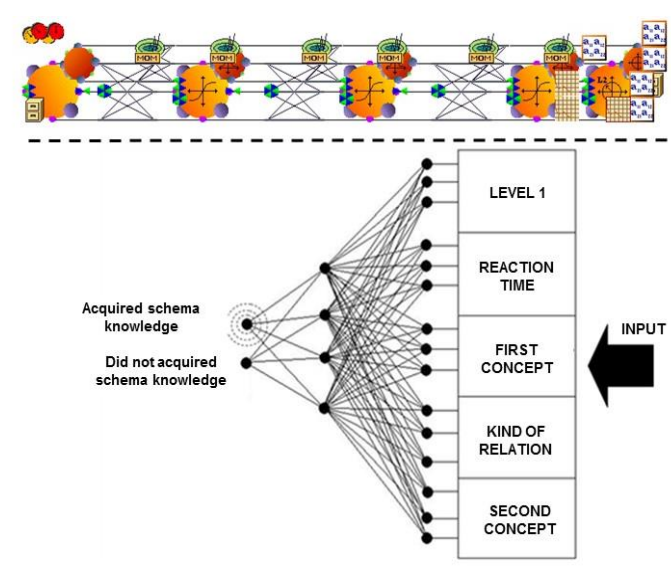

Figure 3. Set of classification simulations

The top part shows a neural net architecture with an input layer unit, two hidden layers, an output layer and an error retro propagator. This system learns to classify recognition times of schema related words by a course for successful and non-successful students.

\section{Results}

As a result of the above lines of research a technology transfer project on education technology called EVCO is being implemented in Mexico City. This system is designed to provide teachers another way to cognitively assess e-learning. For instance, a computer system has been implemented to automatically run semantic priming studies either by Internet or personal computer screens (see Figure 4, middle panel). Soon this capacity will be implemented in iPads and iPhones. The use of these last devices is intended not only to test students for schemata priming on school course concepts but to obtain their mental representation on a school topic by applying the natural semantic technique through them. In turn semantic priming studies are based on a 
database of schemata-related words obtained from teachers and successful students' mental representations. Currently, the database consists only of a few high school and university courses from three different Mexican states but plans are to assimilate course mental representations from different knowledge domains nationwide. School teachers will be empowered by accessing the system and requiring reports of schemata organization (20 indices) and meaning formation from other school courses (see Figure 4, right panel).

In addition, natural semantic network structure metrics (Figure 1) can be obtained from semantic networks. This allows comparison of network growing or network developing through a course. This is relevant for teachers who need to know what course schema concepts acquired more relevance for concept organization after learning (e.g., best concept with in-betweenness centrality [15]. Finally, a teacher can also compare this development with other courses on the same topic.
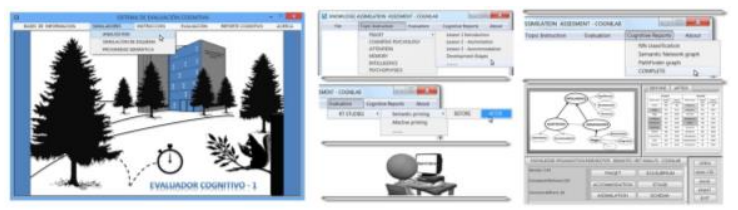

Figure 4. Implementation of computer system for running semantic priming studies

The left panel shows the current EVCO's main computer screen. The middle panel illustrates students taking a semantic priming study from an EVCO menu. A cognitive report can include student classification and a report of a mental representation used to evaluate her/his performance (right panel).

\section{Discussion}

There is a longstanding debate as to whether standard testing is capable of evaluating cognitive learning [22], [23]. Marzano and colleagues [24], [25] [26] have pointed out that standard assessment of learning was not designed to determine, for example, if cognitive abilities or newly-acquired knowledge will remain long term. On the other hand ${ }^{2}$ [27], [28] have found from long-term retention studies that students tend to only retain a reduced knowledge schema of previously tested knowledge.

In regard to acquiring declarative knowledge, mental representation cognitive techniques deal with this assessment problem by directly observing the consequence of learning that is, observing concept acquisition and new schema concept organization of a topic. Even when there is some discussion about which method is best suited to evaluate mental representation due to learning [29], [30], [31], the main problem was the high time cost of applying and processing students' information by using this technique.

Here is an opportunity for computer science to help bring traditional laboratory cognitive science methods to evaluate learning into educational settings. The EVCO system is not intended as a substitute for standard evaluation but as an education technology innovation to obtain information about students' performance that cannot be accessed by traditional methods of evaluation. The computational power in it is used to make a bridge between longknown lab methods to study memory and innovative efforts in education to reduce a tremendous gap between e-instruction development and e-assessment of learning.

As it stands, this system points to new research lines. For instance, it is not known if the schemata priming effect used by the EVCO system applies to all knowledge domains or if teaching efficacy relates to it. More research is demanded.

\section{References}

[1] E.R Steinberg, Computer Assisted Instruction: A synthesis of Theory, Practice and Technology, Lawrence Erlbaum Associates, Publishers, Hillsdale, New Jersey, 1991.

[2] K.S. Hale and K.M. Stanney. Handbook of virtual environments: Design, implementation and applications, Taylor \& Francis: CRC Press, USA, 2014.

[3] M. Keppell, K. Souter and M. Riddle. Physical and Virtual learning spaces in higher education. British Cataloguing in Publication Data, 2012.

[4] J.M. Carroll, Models, theories and frameworks: Toward a multidisciplinary Science (interactive technologies), Morgan and Kaufman Publishers, New York, 2012.

[5] V. Devedzic, Semantic web and education (integrated series in information systems), Springer Science Business Media, New York, 2010.

[6] E. Klopfer, Augmented Learning: Research and design of mobile educational games. Cambridge: MIT Press, 2008 .

[7] J.T. Doswell. "Augmented Learning: Context-Aware Mobile Augmented Reality Architecture for Learning". In the Proceedings of the Sixth IEEE International Conference on Advanced Learning Technologies. IEEE Computer Society, Washington, DC, 2006, pp. 1182-1183.

[8] R.E.O. Lopez, M.G.E. Morales, A.M.I. Hedlefs, T.C.J. Gonzalez, "New empirical directions to evaluate online learning", International Journal of Advances in Psychology, 2014, 3(2), pp 40-47.

[9] C.J, Gonzalez, E.O., Lopez, G.E. Morales, "Evaluating moral schemata learning", International Journal of 
Advances in Psychology, 2013, 2(2), pp. 130-136. ISSN: 2169-494X.

[10] J.G. Figueroa, V.M. Solís and E. Gonzalez, "The possible influence of imaginary upon retrieval and representation in LTM", Acta Psicologica, 1974, 38, pp. 423-428.

[11] J. G., Figueroa, G. E. Gonzales, and V. M. Solis, An approach to the problem of meaning: Semantic networks, Journal of Psycholinguistic Research, 1976, 5(2), pp. 107115 .

[12] Perez, C.N., Hernandez, C. D. Hernandez, B.C. \& Figueroa, N.J. (2012). Model of natural semantic space for ontologies' construction. International Journal of combinatorial Optimization Problems and Informatics, Vol $3,(2), 93-108$.

[13] A. L. Barabási and R. Albert, 1999 "Emergence of scaling in random networks". Science, 286, 509-512.

[14] M. Steyvers and B.J Tenembaum, "The Large-Scale Structure of Semantic Netwoks: Statistic Analysis and a Model of Semantic Grothw", Cognitive Science, 2005, 29, pp. $41-77$.

[15] M.N.I. Bersano, S.E Schaefer and J. J. Bustos. Metrics and models for social networks. In Ajith Abraham, Aboul-Ella Hassanien [Eds]. Computational Social Networks: Tools, Perspectives and Applications, Springer Verlag, London, 2012, pp. 115-142.

[16] D. E. Rumelhart, P. Smolensky, J. L. McClelland and G.E. Hinton, Schemata and sequential thought processes. In: J.L., McClelland, D. E. Rumelhart, and the PDP research group. Parallel distributed processing: Explorations in the microstructure of cognition. Volume 2: Psychological and biological models. Massachusetts: MIT Press, 1986.

[17] R. E. O. Lopez and J. Theios, "Semantic Analyzer of Schemata Organization (SASO)", Behavior Research Methods, Instruments, \& Computers, 1992, 24(2), pp. $277-$ 285.

[18] R. E. O. Lopez, Schematically related word recognition: Ph.D. dissertation abstract. Michigan: UMI Dissertation Abstracts International, 1996.

[19] R. E. O. Lopez, \& J. "Theios. Semantic Analyzer of Schemata Behavior (SASO)". Presented at "The Society for Computers in Psychology. Twenty-first Annual Conference". San Francisco, 1991.

[20] R.E.O. Lopez, M.V.M. Padilla and N.M.C. Rodriguez, Connectionist schemata based behavior based on subject conceptual definitions: The role of inhibitory mechanisms. 4th International Conference on Memory. University of New South Wales, Sydney, Australia, 2006.
[21] R.E.O. Lopez and J. Theios, "Single word schemata priming: a connectionist approach". Paper presented at the 69th Annual Meeting of the Midwestern Psychological Association, Chicago, IL, 1997.

[22] J., Pellegrino, N., Chudowsky and R. Glaser. Knowing what students know: The science and design of educational assessment. National Academy Press, Washington, DC, 2001.

[23] P. Nichols and B. Sugrue, "The lack of fidelity between cognitively complex constructs and conventional test development practice", Educational Measurement: Issues and Practice, 1999, 18, 18-29.

[24] R. J. Marzano, Lessons from the field about outcomebased performance assessments, Educational Leadership, March 1994, pp. 44-50.

[25] R.J., Marzano, D.J. Pickering and R.S. Brandt, "Integrating instructional programs through dimensions of learning", Educational Leadership, February 1990, pp. 1724.

[26] R. J. Marzano and A. Costa, "Question: Do standardize tests measure general cognitive skills?" Answer: No. Educational Leadership, May 1998, pp. 6671.

[27] M.A. Conway, G. Cohen and N. Stanhope, "On the Very Long-Term Retention of Knowledge Acquired Through Formal Education: Twelve Years of Cognitive Psychology". Journal of Experimental Psychology: General, 1991, 120, pp. 395-409.

[28] M.A Conway, G. Cohen and N. Stanhope, "Very long term memory for knowledge acquired at school and university", Applied Cognitive Psychology, 1992, 6, pp. 467-482.

[29] R.B. Clariana and R. Koul, "The effects of learner prior knowledge when creating concept maps from a text passage", International Journal of Instructional Media, 2008, 35(2), pp. 177-184.

[30] P. Gonzalvo, J.J. Cañas and M.T. Bajo. "Structural representation in knowledge acquisition", Journal of Educational Psychology, 1994, 86, 601-616.

[31] T.E. Goldsmith, P.J. Johnson \& W.H. Acton, "Assessing structural knowledge", Journal of Educational Psychology, 1991, 83, pp. 88-96. 